ature (9-21 K) using electrochemically etched tungsten wires for analyzing tips, in order to record the diffusion of single copper adatoms on the close-packed $\mathrm{Cu}(111)$ substrate. They prepared their samples by evaporating 0.01 monolayer of copper at $15 \mathrm{~K}$, a temperature at which single copper atoms are mobile, on $\mathrm{Cu}(111)$ substrates that were previously cleaned by several sputter and anneal cycles.

The researchers analyzed STM images showing two $\mathrm{Cu}$ adatoms separated by distances of up to $70 \AA$. More than 65,000 $\mathrm{Cu}$ spacing distances from a total of 3400 images were analyzed. The researchers established an oscillatory behavior of the potential energy, with a periodicity of $\lambda_{F} / 2$, and an envelope that decays as $\sim 1 / d^{2}$ for large separation $d$. The method used to determine the interaction potential was to extract it from the measured pair distribution, obtained from the timedependence of the distance between two adatoms, correcting for geometrical effects inherent to the measuring process. The discrepancies between their experimental results and the previous theoretical descriptions of the phenomenon will help to re-evaluate the assumptions made as well as neglected terms in theoretically describing the potential energy between adatoms, and will also help to understand the growth of $\mathrm{Cu}$ on $\mathrm{Cu}(111)$ at low temperatures.

Claudiu Muntele

\section{Nucleation and Growth Mechanism Causes Switching of Exchange Bias in Double- Superlattice System}

At interfaces of ferromagnetic and antiferromagnetic films, exchange coupling results in a process called "exchange bias," in which the ferromagnetic hysteresis loop shifts. Investigators at Argonne National Laboratory, studying the exchange bias in a "double-superlattice" system, have observed reversal of direction ("switching") of the exchange bias. They attribute the switching mechanism to a breakdown into domains of the antiferromagnetically coupled (AF) superlattice by nucleation and growth, followed by complete reversal of magnetization in the layers. They reported this result, which challenges the uniform rotation model, in the October 2 issue of Applied Physics Letters.

Using the $\mathrm{Fe} / \mathrm{Cr}(211)$ system for their study, researchers S.G.E. te Velthuis, J.S. Jiang, and G.P. Felcher combined a ferromagnetically coupled $(\mathrm{F})$ superlattice consisting of $50 \AA \mathrm{Fe}$ and $20 \AA \mathrm{Cr}$ with an $\mathrm{AF}$ superlattice of $14 \AA$ Fe and $11 \AA \mathrm{Cr}$ to form a double superlattice with the layer sequence $[\mathrm{Fe}(50 \AA) / \mathrm{Cr}(20 \AA)]^{\mathrm{F}}{ }_{5} /[\mathrm{Fe}(14 \AA) /$ $\mathrm{Cr}(11 \AA)]^{\mathrm{AF}}{ }_{20}$. A $20-\AA$ layer of $\mathrm{Cr}$ was sandwiched between the F and AF superlattices to provide a ferromagnetic intersuperlattice coupling. An artificial exchange bias (uniaxial anisotropy) was built into the system by epitaxially growing the sample onto a single-crystal $\mathrm{MgO}(110)$ substrate.

Magneto-optic Kerr effect measurements were performed around two critical values ( -406 Oe and $-447 \mathrm{Oe})$ of the turning field in the field loop, $\mathrm{H}_{\min }$. The hysteresis loop obtained for the -406 Oe measurements is narrow, with a bias around $-38.5 \mathrm{Oe}$; the magnetization in the $\mathrm{F}$ superlattice reverts to its original orientation at -33.6 Oe. However, with $\mathrm{H}_{\min }$ at $-447 \mathrm{Oe}$, the $\mathrm{F}$ superlattice magnetization does not revert to its original orientation until $40.7 \mathrm{Oe}$, indicating that the AF superlattice has reversed its direction. Polarized neutron reflectivity measurements confirm the reversal of the AF superlattice. The fact that the bias direction switches at a value of $-447 \mathrm{Oe}$, which is much lower than the $14-\mathrm{kOe}$ field required to saturate the AF superlattice, or the 2-kOe field needed to initiate spinflop transitions, indicates that a different mechanism is at work. A nucleation and growth scenario is consistent with these results; further work is under way to determine the field-dependence of the magnetic layer structure more precisely.

TIM PALUCKA

\section{Thin Films of $\alpha-\mathrm{Al}_{2} \mathrm{O}_{3}$ Result from the Use of an Alternative Anhydrous Solution}

Thin films of crystalline $\alpha-\mathrm{Al}_{2} \mathrm{O}_{3}$ are obtained through an innovative sol-gel process developed by Naoufal Bahlawane and Tadahiko Watanabe at the Kyushu National Industrial Research Institute in Japan. This process has the advantage of reducing the transformation temperature for $\alpha-\mathrm{Al}_{2} \mathrm{O}_{3}$, gaining more control over the final conditions of particle shape and size. Another advantage of this process is the accomplishment of a direct conversion to $\alpha-\mathrm{Al}_{2} \mathrm{O}_{3}$ with the use of an anhydrous

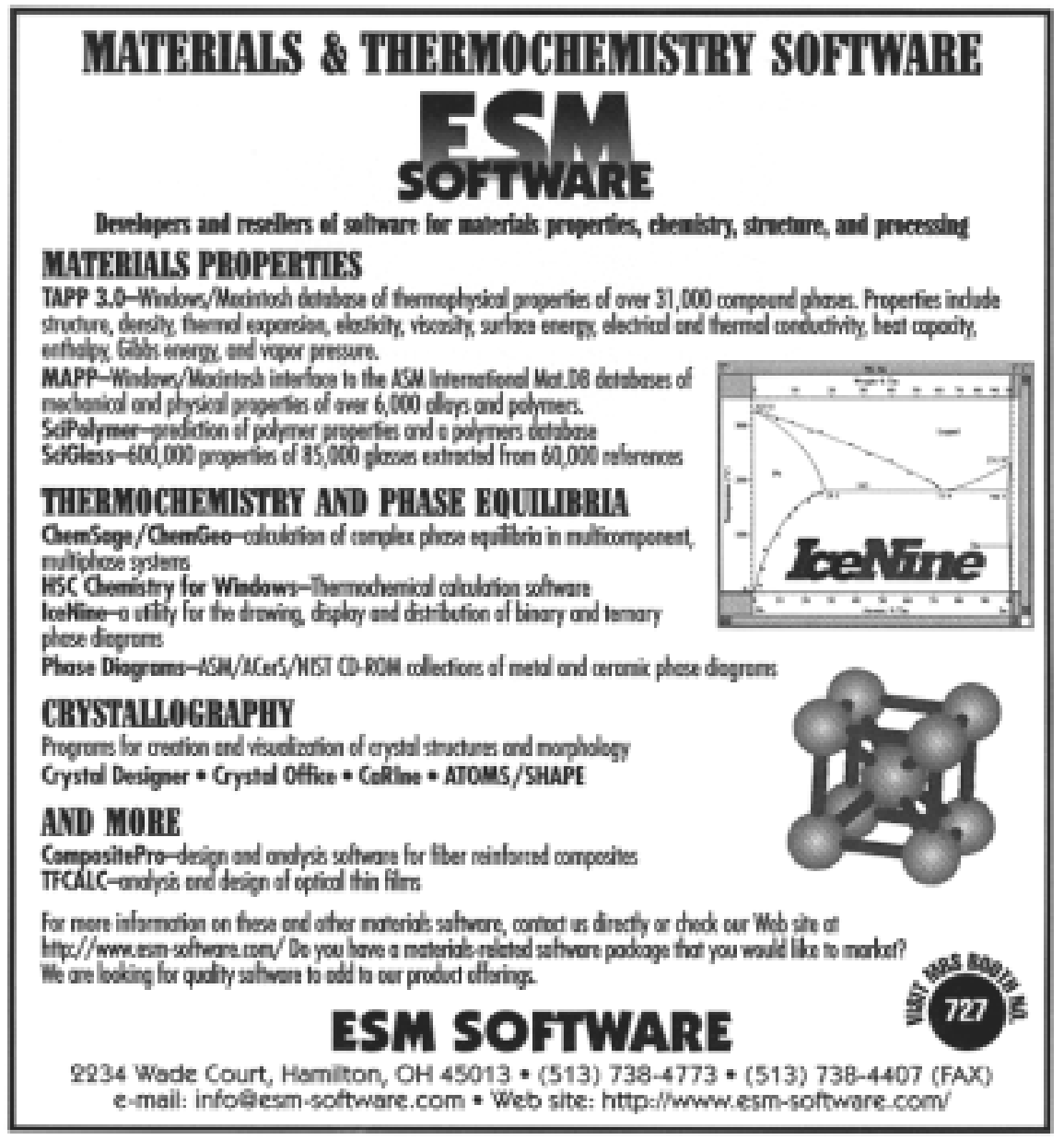

Circle No. 13 on Inside Back Cover 